\title{
The Effects of Resistance Exercise in End Stage Renal Disease Patients Undergoing Hemodialysis: A Systematic Meta-Analysis
}

\author{
Penpayome Sakorn ${ }^{1}$ and Pachanat Nanthaitaweekul ${ }^{1,2, *}$ \\ ${ }^{1}$ Faculty of Nursing, Chulalongkorn University, Bangkok 10330, Thailand \\ ${ }^{2}$ Developing of Caring Guidelines among to Patients with Cardiovascular Disease from Hospital to \\ Community Research Group, Chulalongkorn University, Bangkok 10330, Thailand
}

('Corresponding author's e-mail: pachanut.t@chula.ac.th)

Received: 20 April 2021, Revised: 8 June 2021, Accepted: 20 June 2021

\begin{abstract}
End-stage renal disease (ESRD) is a global public health problem as same as in Thailand the number of patients undergoing hemodialysis treatment is getting higher every year. In frequently cases has many impacts and affect the reduction of functional conditions. The objective of this study is to conduct a systematic review and meta-analysis of research into the effects of resistance exercise on end stage renal disease patients undergoing hemodialysis and to analyze the effect size of resistance exercise on patients. A systematic literature search was conducted using the database of Science direct, Wiley Online Library, Scopus, PubMed, EBSSCO host and the Thai-Journal Citation Index Center, looking for randomized controlled trials between 2009 and 2020. Combined effect sizes were calculated using Review Manager Version 5.3. Eight articles met the inclusion criteria and in the effect size analysis. Results showed that resistance exercise improved the ability to function and increased muscle strength, improved body composition and quality of life in chronic renal failure patients undergoing hemodialysis treatment.
\end{abstract}

Keywords: Resistance exercise, Intervention, End stage renal disease, Hemodialysis, Systematic review

\section{Introduction}

Chronic Renal Failure (CRF) is a global public health problem experienced by $11-13 \%$ of the global population [1]. A SEEK study conducted by the Nephrology Society of Thailand in 2009 found $17.5 \%$ of the country's population suffered from chronic renal disease. Furthermore, the number of patients receiving kidney replacement therapy increased rapidly from 1,053 to 13,308 in 2010, driving estimated related healthcare costs to 3,500 million baht per year [2]. End-stage renal disease is the prognosis of permanent loss of kidney function which decreases the excretion of waste from the body due to imbalances between water and minerals. Patients cannot be cured, so kidney replacement therapy is essential. Methods include hemodialysis, peritoneal dialysis and kidney transplant [3]. Patients with end-stage renal disease (ESRD) undergoing hemodialysis experience a range of physical, mental, social, and spiritual discomforts. In terms of the physical impact, the patient had needs to face various symptoms and conditions such as bone and joint pain, muscular weakness, lack of energy and low self-assistance ability [4]. Psychologically, they are prone to anxiety and depression because of the change in their social life and environment [5]. Patients feels uncertain, as diagnosis and treatment entails change in relationships with other people [6,7]. In addition, they feel that they are a burden to their families and society because need help with the activities of daily life or able to do short-term activities [8]. This was consistent with Bossola and Enrico's [9] findings that patients undergoing hemodialysis were prevented from doing $26 \%$ of the Activities of Daily Life (ADL), while the ability to do Intensive Activities of Daily Life (IADL) was reduced by $48 \%$. Moreover, Kim et al. [8] found that patients undergoing hemodialysis were $60-70 \%$ less able to perform routine tasks and physical functions than healthy adults. Therefore, the meta-analysis study related with exercise program is necessary for developing the self-efficacy promoting and resistance training program in End Stage Renal Disease (ESRD) patients receiving hemodialysis (This is our research project in the future).

Research studies have been conducted on a number of exercise programs for the end stage renal failure patients undergoing hemodialysis. Both aerobic and resistance exercise can reduce the above complications [10], increasing muscle strength [11] and the ability to perform daily activities, reducing depression and the 
common symptom of restless legs, as well as generally increasing quality of life [11-13]. However, there is not yet a literature review looking into effective method that works best for patients undergoing hemodialysis. Therefore, this literature review and meta-analysis aims to collate the characteristics, methods and effect size to be development of resistance exercise programs on muscle strength, physical ability, physical performance and quality of life.

Resistance exercise refers to anaerobic exercise that does not increase oxygen use during exertion. As the exercise increases muscle exertion towards full force, it increases muscle strength, hypertrophy and mass [14-16]. Resistance exercise can be divided into 2 types: 1) Isotonic exercise, which involves contraction of the muscles; the length of the muscle changes and moves objects such as dumbbells, barbells, elastic resistance bands or the subject's own body weight. 2) Isometric exercise, which does not involve the movement of the joints; rather, the muscles are fixed in length, tense and tightened against resistance, such as in wall pushing and planking [16].

The American College of Sports Medicine [15] recommends that resistance exercise is performed 2 - 3 times a week at light to medium intensity (RPE Borg's scale $=11-15$ ) and repeated 8 - 12 times per set, which can be adjusted according to ability, and can use a variety of equipment such as free weight dumbbells, resistance machines, resistance bands or tubes or body weight.

Resistance exercise has a positive effect on the health of end-stage renal failure patients undergoing hemodialysis. The benefits to patients were a 18 - $75 \%$ reduction in energy and protein loss (Protein Energy Wasting; PEW), using mechanisms to balance protein synthesis and breakdown, increase the retention of protein in the muscles. Increased muscle mass can also reduce inflammatory processes in the body $[17,18]$, and inflammatory markers are reduced by inhibiting the release of Tumor Necrosis Factor Alpha (TNF- $\alpha$ ), which is responsible for provoking inflammation [17]. The synthesis of more proteins and strengthens the patient's muscles and can increase functional capacity [17-19]. The increase in muscle fibers increases the flow of oxygen to muscles and causes increased glucose oxidation [20]. The increasing efficiency of muscle function allows patients to do more daily activities on their own $[18,19]$. Also, increased muscle mass, or what us referred to as skeletal muscle hypertrophy, promotes muscle energy turnover [21]. The resultant increases in bone density and reduced bone loss from bone resorption, in addition decrease parathyroid hormone levels. Furthermore, they inhibit the negative effects of parathyroids (iPTH) that affects bone remodeling, maintains bone quality and strengthens bone structure in patients receiving hemodialysis [22]. In addition, resistance exercise increases the overall strength and ability of the patient [18], allowing them to participate in social activities [23].

\section{Materials and methods}

Literature search

This systematic review collates relevant studies registered and published in Thai or English from 2009 to 2020 with research methodology including meta-analysis and effect size calculations. Articles came from online libraries such as Science Direct, Wiley Online Library, Scopus, PubMed (US Library of Medicine and National Institutes of Health), EBSSCO host and Thai-Journal Citation Index Centre between 2009 2020 Each article was selected and screened by title and abstract. Full-text articles were retrieved and scanned when abstracts did not give enough data for a decision to be made. Article selection was carried out by 2 researchers. Any conflict was solved by a third reviewer.

\section{Eligibility criteria}

The research characteristics using descriptive statistics. For instance, analyzing the effect size and bias risk with Review Manager 5.3 [24] to compute continuously variable data with standardized mean differences; SMD and $95 \%$ of the confidence interval (CI). Furthermore, analyzing the effect according to Cohen's criteria: effect sizes higher than 0.8 , between 0.8 and 0.2 , and less than 0.2 , indicate large, medium, and small effects, respectively [25]. Heterogeneity was obtained by Cochrane's Q test and $\mathrm{I}^{2}$ statistics. If there was no significance in the study $\left(p<0.10\right.$ and $\left.\mathrm{I}^{2}>50 \%\right)$, the data was combined with a fixed-effect model or if there were significant a random effect model will be applied.

From Table 1, in order to structure the eligibility criteria, the PICOS approach was used, including population, intervention, comparator, outcome and study design. All of articles were randomized controlled trials between 2009 and 2020. 
Table 1 Eligibility criteria by category (PICOS) in end stage renal disease patients with hemodialysis.

\begin{tabular}{cl}
\hline Category & \multicolumn{1}{c}{ Eligibility criteria } \\
\hline Population & $\begin{array}{l}\text { End stage renal disease patient, Chronic kidney disease stage 5, } \\
\text { Hemodialysis patient }\end{array}$ \\
\hline Intervention & Progressive resistance exercise, Resistance exercise \\
\hline Comparator & Control group \\
\hline Outcome & BMI, Physical performance, QOL, Muscle strength \\
\hline Study design & Randomized controlled trials, Cluster randomized controlled trials \\
\hline
\end{tabular}

Each sample was a randomized controlled trial (RCT) on resistance exercise in end stage renal disease patients undergoing hemodialysis. Inclusion criteria were as follows: 1) each article was in Thai and English, 2) each study focuses on patient with end-stage renal failure undergoing hemodialysis. 3) the sample group must be over 18 years of age, and 4) the study is quantitative and involves randomized controlled trials (RCT).

\section{Included meta-analyses}

Flow diagram of the search and selection process is presented in Figure 1. The initial search resulted in 306 publications. Removal of duplicates resulted in 284 remaining articles. After screening the publications by title and abstract, 210 articles did not meet the criteria, mainly because they did not employ a meta-analysis. The remaining 36 articles were read in full text and at this stage 28 article were excluded, mostly because they no relevant outcome $(n=9)$, not RCT $(n=18)$ and not English $(n=1)$ Finally, 8 metaanalyses were included in this study.

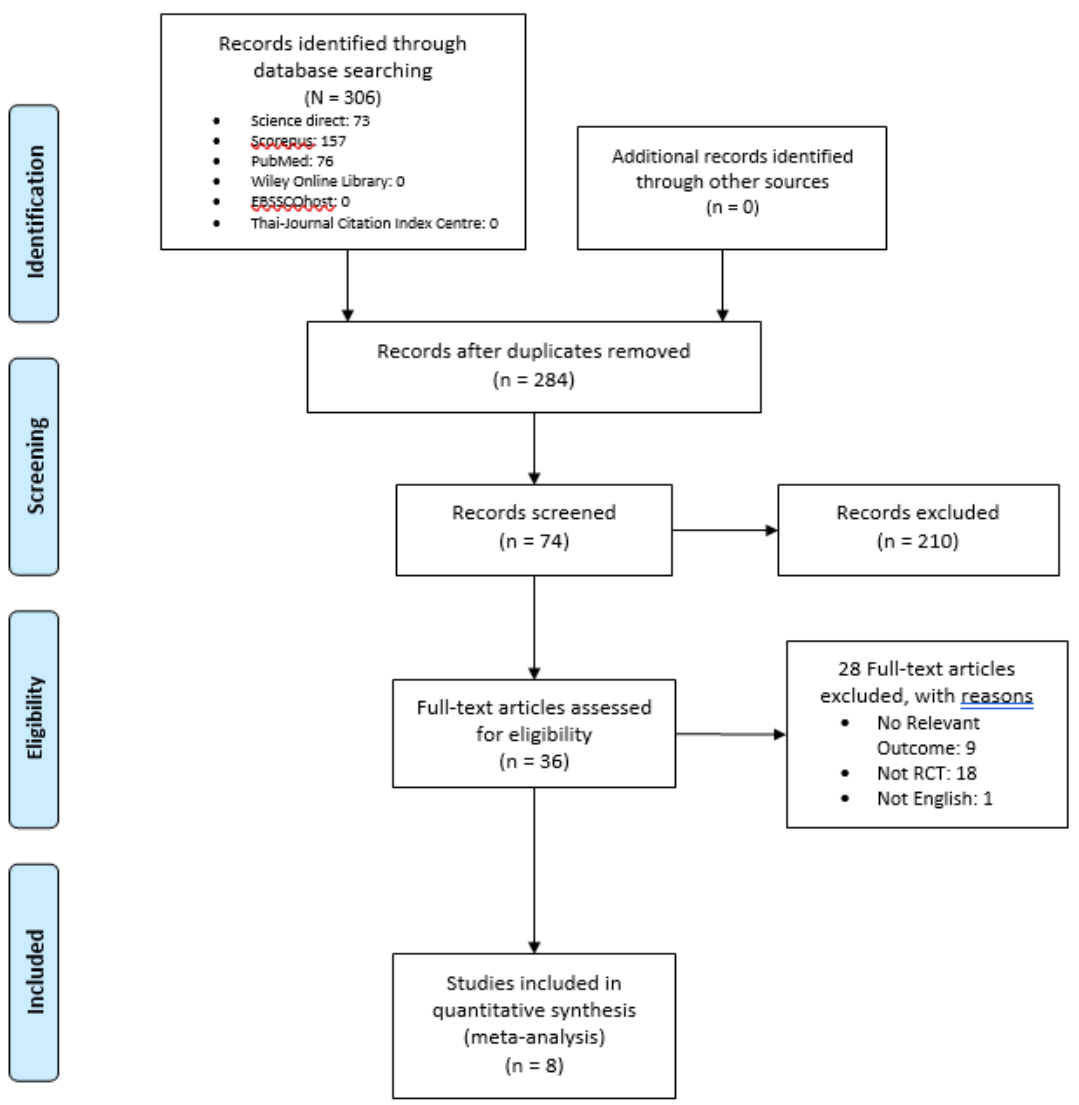

Figure 1 Flow diagram of study selection to be used in research analysis and synthesis inclusion 4 steps: Identification, screening, eligibility and include. 


\section{Publication bias}

No research came from an unpublished resource.

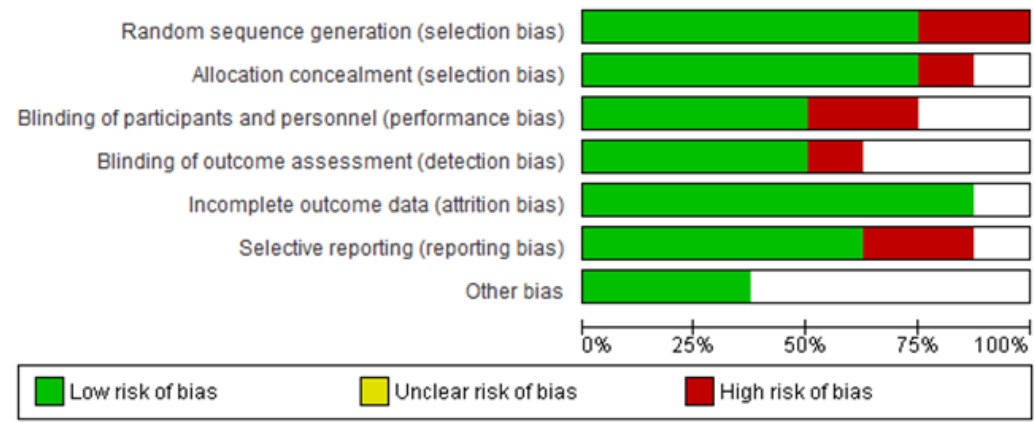

Figure 2 Risk of bias graph: Review authors' judgments about each risk of bias item presented as percentages across all included studies.

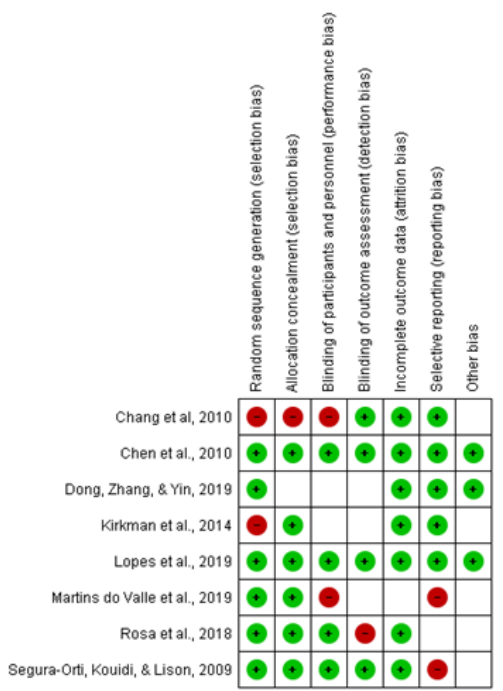

Figure 3 Risk of bias summary: Review authors' judgments about each risk of bias item for 8 studies will also find that risk of bias was low.

\section{Risk of bias}

From Figures 2 and 3: Assessment of selected researcher's percentage risk of bias, most studies are at low risk of bias that show more than $50 \%$ (Figure 2) including: Random sequence generation (75\%), Allocation concealment $(75 \%)$, blinding of participant and personnel $(50 \%)$, blinding of outcome assessment (50\%), Incomplete outcome data (90\%) and selective report (60\%). In addition, from Figure 3 , the 8 studies will also find that risk of bias was low by 6 studies reported the random allocation and used the allocation concealed in low risk of bias. Only 4 studies reported that blinding of participant and personnel outcome assessment also blinding of outcome assessment. Five studies with selection reporting. Moreover, there were 7 studies reported that there was low risk of bias in incomplete outcome data.

\section{Data extraction}

Data on relevant study characteristics and effect sizes (standardized mean difference (SMD) from meta-analytic comparisons were extracted by 2 researches. Information was extracted on the number of RCTs. Data on heterogeneity present in meta-analytic comparisons was also obtained. Extracted study characteristics were based on PICOS (participants, interventions, comparators, outcomes and study design) 
criteria. The descriptions given in Table 2 were extracted from the authors' reports on the individua studies as well as from data reported within individual studies.

\section{Results and discussion}

\section{Participants and characteristics of 8 studies}

In Table 2 are the characteristics of each study obtained from the systematic review. There are a total of 8 research studies, with 3 from Brazil [26-28] and 1 each from the United Kingdom (UK) [19], Taiwan [29], China [30], Spain [31] and the United States [14]. From a total of 341 hemodialysis patients, 76 patients were the largest sample from a single study [29], while 23 was the smallest [19]. The sample group age range was between 49 - 71 years old [30]. Participants undertook light aerobic exercise in 2 studies [28$31]$, muscle stretches in 4 studies [14,19,26,27] and unknown exercises in 1 study [26,29,31]

As shown in Table 2, the majority of resistance exercise programs used small devices such as free weights, weighted ankle cuffs and elastic resistance bands [14,26,30,31]. In terms of larger devices, 1 study investigated the effects of leg ergometry [29] and 1 the leg press [19], and there was 1 study that did not identify the devices used in its resistance exercises [28].

Resistance exercises were undertaken 3 times a week in all studies except 1, in which patients only exercised twice per week [14]. The duration of each exercise session was around 30 - 50 min [28,29], with most exercises repeated 8 - 15 times per set, for 2 - 3 set each session. Only 1 study performed 10 times $\times 10$ cycles [30] and the majority of programs lasted 12 weeks [19,26-28,30]. However, one program lasted 6 months [31], one 24 weeks [14] and one had a shorter duration at 8 weeks [29].

Most exercise was of light intensity [14,27,29,30], while 2 programs were of light to moderate intensity exercise [19,31], one of moderate to strong intensity [26], and one article did not specify intensity [28]. Most resistance exercise was started during the hemodialysis process, with 4 studies investigating exercise during the first $2 \mathrm{~h}$ of dialysis [14,26,27,31], one within the first hour of dialysis [29], and 2 that did not clearly specify exercise start time [19,30]. The remaining study divided exercise into 2 sessions: one during the waiting period for dialysis and another during the dialysis period itself [28]. All exercise was undertaken under the supervision of either a specialist or nurse.

Table 2 The characteristics of each study obtained from 8 research publication of resistance exercise in end stage renal failure and hemodialysis patients.

\begin{tabular}{|c|c|c|c|c|c|c|c|c|c|}
\hline Authors & Country & $\mathbf{N}$ & Age & $\begin{array}{l}\text { Drop out } \\
(\mathbf{n}, \%)\end{array}$ & $\begin{array}{l}\text { Control } \\
\text { group }\end{array}$ & $\begin{array}{c}\text { Resistance } \\
\text { training } \\
\text { group }\end{array}$ & $\begin{array}{l}\text { Training: } \\
\text { FIIT*** }\end{array}$ & Duration & Outcome \\
\hline \multirow[b]{2}{*}{$\begin{array}{c}\text { Segura- } \\
\text { Orti et al. } \\
\text { [31] }\end{array}$} & \multirow[b]{2}{*}{ Spain } & $\mathrm{I}^{*}: \mathrm{n}=17$ & $\begin{array}{c}53.50 \pm \\
18.00\end{array}$ & $\begin{array}{c}2 \\
(10.53 \%)\end{array}$ & \multirow[b]{2}{*}{$\begin{array}{c}\text { low-intensity } \\
\text { aerobic } \\
\text { training }\end{array}$} & \multirow[b]{2}{*}{$\begin{array}{c}\text { ankle } \\
\text { weight, } \\
\text { elastic band. }\end{array}$} & \multirow{2}{*}{$\begin{array}{l}\text { F: } 3 \text { times/week } \\
\text { I: } 15 \text { Repetitions } \\
\text { (Rep.) } \times 3 \text { Sets } \\
\text { I: low-moderate } \\
\text { intensity (Borg's } \\
\text { scale } 12-15) \\
\text { T: } 6 \text { months }\end{array}$} & \multirow{2}{*}{$\begin{array}{l}\text { Intradialytic: } \\
\text { The first } 2 \mathrm{~h} \\
\text { of } \\
\text { Hemodialysis } \\
\text { (HD) sessions }\end{array}$} & \multirow{2}{*}{$\begin{array}{c}\text {-Physical } \\
\text { performance } \\
\text {-Unknow exercise }\end{array}$} \\
\hline & & $C^{*}: n=8$ & $\begin{array}{c}60.10 \pm \\
16.90\end{array}$ & $\begin{array}{c}0 \\
(0.00 \%)\end{array}$ & & & & & \\
\hline \multirow[b]{2}{*}{$\begin{array}{c}\text { Chen et al. } \\
{[14]}\end{array}$} & \multirow[b]{2}{*}{ USA } & $\mathrm{I}: \mathrm{n}=25$ & $71.1 \pm 12.6$ & $\begin{array}{c}3 \\
(12 \%) \\
\end{array}$ & \multirow[b]{2}{*}{$\begin{array}{l}\text { stretching } \\
\text { exercises } \\
\text { with light } \\
\text { resistance } \\
\text { bands }\end{array}$} & \multirow[b]{2}{*}{$\begin{array}{l}\text { ankle } \\
\text { weight } \\
+ \text { free } \\
\text { weight }\end{array}$} & \multirow[b]{2}{*}{$\begin{array}{c}\text { F: } 2 \text { times/week } \\
\text { I: } 8 \text { Rep. } \times 2 \text { sets. } \\
\text { I: low intensity } \\
\text { (Borg's scale }= \\
2 \text { - } 4 \text { of } 10 \text { ) or } 60 \\
\% \text { Maximum } \\
\text { Heart Rate } \\
\text { T: } 24 \text { weeks }\end{array}$} & \multirow[b]{2}{*}{$\begin{array}{l}\text { Intradialytic: } \\
\text { During the } \\
\text { second hour } \\
\text { of HD } \\
\text { sessions. }\end{array}$} & \multirow{2}{*}{$\begin{array}{c}\text { - Physical } \\
\text { performance } \\
\text { - Muscle strength } \\
\text { - Body } \\
\text { composition: Lean } \\
\text { and fat mass } \\
\text { - Quality of Life } \\
\text { (QoL): Physical } \\
\text { component } \\
\text { - Physical Activity }\end{array}$} \\
\hline & & $C: n=25$ & $66.9 \pm 13.4$ & $\begin{array}{c}3 \\
(12 \%)\end{array}$ & & & & & \\
\hline \multirow[b]{2}{*}{$\begin{array}{l}\text { Chang et } \\
\text { al. [29] }\end{array}$} & \multirow[b]{2}{*}{ Taiwan } & $\mathrm{I}: \mathrm{n}=39$ & $\begin{array}{c}50.80 \pm \\
10.72 \\
\end{array}$ & $\begin{array}{c}3 \\
(7.7 \%) \\
\end{array}$ & \multirow[b]{2}{*}{ N/A } & \multirow[b]{2}{*}{$\begin{array}{c}\text { leg } \\
\text { ergometry }\end{array}$} & $\begin{array}{c}\mathrm{F}: 3-4 \\
\text { times/week } \\
\text { I. } 30 \mathrm{~min}\end{array}$ & \multirow{2}{*}{$\begin{array}{l}\text { Intradialytic: } \\
\text { During the } \\
\text { first hour of } \\
\text { HD sessions }\end{array}$} & \multirow{2}{*}{$\begin{array}{c}\text { - Fatigue } \\
\text { - Physical Activity } \\
\text {-unknown } \\
\text { exercise }\end{array}$} \\
\hline & & $\mathrm{C}: \mathrm{n}=37$ & $52.00 \pm 8.70$ & $\begin{array}{c}2 \\
(5.4 \%)\end{array}$ & & & $\begin{array}{c}\text { I: low intensity } \\
\text { (Borg's scale }= \\
12-13 \text { ) } \\
\text { T: } 8 \text { weeks. }\end{array}$ & & \\
\hline \multirow{2}{*}{$\begin{array}{l}\text { Kirkman } \\
\text { et al. }[19]\end{array}$} & \multirow{2}{*}{ UK } & $\mathrm{I}: \mathrm{n}=12$ & $\begin{array}{c}48.00 \pm \\
18.00\end{array}$ & $\begin{array}{c}3 \\
(25 \%)\end{array}$ & \multirow{2}{*}{$\begin{array}{l}\text { stretching } \\
\text { exercise }\end{array}$} & \multirow{2}{*}{$\begin{array}{l}\text { leg press } \\
\text { exercise. }\end{array}$} & \multirow{2}{*}{$\begin{array}{c}\text { F: } 3 \text { times/week } \\
\text { I: } 10-15 \text { Rep. } \times 3 \\
\text { sets }\end{array}$} & \multirow{2}{*}{$\begin{array}{l}\text { Intradialytic: } \\
\text { Duration: } \\
\text { N/A }\end{array}$} & \multirow{2}{*}{$\begin{array}{c}\text { - Muscle strength } \\
\text { - Anabolic } \\
\text { response }\end{array}$} \\
\hline & & $\mathrm{C}: \mathrm{n}=11$ & $\begin{array}{c}58.00 \pm \\
15.00\end{array}$ & $\begin{array}{c}0 \\
(0 \%)\end{array}$ & & & & & \\
\hline
\end{tabular}




\begin{tabular}{|c|c|c|c|c|c|c|c|c|c|}
\hline Authors & Country & $\mathbf{N}$ & Age & $\begin{array}{l}\text { Drop out } \\
(\mathbf{n}, \%)\end{array}$ & $\begin{array}{l}\text { Control } \\
\text { group }\end{array}$ & $\begin{array}{c}\text { Resistance } \\
\text { training } \\
\text { group }\end{array}$ & $\begin{array}{l}\text { Training: } \\
\text { FIIT*** }\end{array}$ & Duration & Outcome \\
\hline & & & & & & & $\begin{array}{l}\text { I: Low-Moderate } \\
\text { intensity (Borg's } \\
\text { scale < 15) } \\
\text { T: } 12 \text { weeks. }\end{array}$ & & \\
\hline \multirow[b]{2}{*}{$\begin{array}{l}\text { Rosa et al. } \\
\text { [28] }\end{array}$} & \multirow[b]{2}{*}{ Brazil } & I: $n=28$ & $\begin{array}{c}54.49 \pm \\
11.97 \\
\end{array}$ & $\begin{array}{c}2 \\
(6.67 \%) \\
\end{array}$ & \multirow{2}{*}{$\begin{array}{l}\text { very low } \\
\text { intensity } \\
\text { exercise } \\
\text { without load } \\
\text { and } \\
\text { progression }\end{array}$} & \multirow{2}{*}{$\begin{array}{l}11 \text { exercises } \\
\text { progressive } \\
\text { resistance } \\
\text { training. } \\
\text { Equipment: } \\
\text { N/A }\end{array}$} & \multirow{2}{*}{$\begin{array}{c}\text { F: } 3 \text { times/week. } \\
\text { I: } 15-20 \text { Rep. } \\
\times 2 \text { sets, } 40-50 \\
\text { min. } \\
\text { I: N/A } \\
\text { T: } 12 \text { weeks }\end{array}$} & \multirow{2}{*}{$\begin{array}{l}2 \text { sessions; } \\
\text { before the } \\
\text { HD sessions } \\
\text { and } \\
\text { during the } \\
\text { HD sessions. } \\
\text { Duration: } \\
\text { N/A }\end{array}$} & \multirow{2}{*}{$\begin{array}{c}\text { - Body } \\
\text { composition: leg } \\
\text { lean mass } \\
\text { - Physical } \\
\text { performance } \\
\text { - Muscle strength }\end{array}$} \\
\hline & & $C: n=24$ & $\begin{array}{c}57.10 \pm \\
16.20\end{array}$ & $\begin{array}{c}5 \\
(17.24 \%)\end{array}$ & & & & & \\
\hline \multirow[b]{2}{*}{$\begin{array}{l}\text { Dong et } \\
\text { al. }[30]\end{array}$} & \multirow[b]{2}{*}{ China } & $\mathrm{I}: \mathrm{n}=21$ & $\begin{array}{c}59.0 \\
(32.5,66.5) \\
\end{array}$ & $\begin{array}{c}2 \\
(8.70 \%) \\
\end{array}$ & \multirow[b]{2}{*}{$\begin{array}{c}\text { routine } \\
\text { hemodialysis } \\
\text { care }\end{array}$} & \multirow[b]{2}{*}{$\begin{array}{l}\text { free weight, } \\
\text { the ankle } \\
\text { weight, the } \\
\text { elastic ball }\end{array}$} & \multirow{2}{*}{$\begin{array}{c}\text { F: } 3 \text { times/week } \\
\text { I: } 10 \text { Rep. } \times 10 \\
\text { cycles } \\
\text { I: low intensity } \\
\text { (Borg's scale }< \\
15 \text { or }<80 \% \text { of } \\
\text { HRmax) } \\
\text { T: } 12 \text { weeks. }\end{array}$} & \multirow[b]{2}{*}{$\begin{array}{l}\text { Intradialytic: } \\
\text { Duration: } \\
\text { N/A }\end{array}$} & \multirow{2}{*}{$\begin{array}{c}\text { - Muscle strength } \\
\text { - Physical activity } \\
\text { - serum albumin } \\
\text { - Kt/V } \\
\text { - Inflammatory } \\
\text { modulation }\end{array}$} \\
\hline & & $C: n=20$ & $\begin{array}{c}62.5 \\
(50.5,70.0)\end{array}$ & $\begin{array}{c}1 \\
(4.76 \%)\end{array}$ & & & & & \\
\hline \multirow{3}{*}{$\begin{array}{c}\text { Lopes } \\
\text { et al. [26] }\end{array}$} & \multirow{3}{*}{ Brazil } & $\begin{array}{l}\text { HLG: } \\
\mathrm{n}=14\end{array}$ & $48.1 \pm 10.8$ & $\begin{array}{c}13 \\
(48.15 \%)\end{array}$ & \multirow{3}{*}{$\begin{array}{l}\text { weekly } \\
\text { stretching } \\
\text { sessions } \\
\text { with elastic } \\
\text { bands }\end{array}$} & \multirow{3}{*}{$\begin{array}{c}\text { elastic } \\
\text { bands and } \\
\text { ankle } \\
\text { weights }\end{array}$} & \multirow{3}{*}{$\begin{array}{c}\text { F: } 3 \text { times/week. } \\
\text { I: } 8 \text { - } 16 \text { Rep. } \times 2 \\
-3 \text { sets. } \\
\text { (HLG, } 8-10 \\
\text { rep.), (MLG, } 16 \text { - } \\
18 \text { rep.) } \\
\text { I: Moderate- } \\
\text { High intensity } \\
\text { T: } 12 \text { weeks }\end{array}$} & \multirow{3}{*}{$\begin{array}{l}\text { Intradialytic: } \\
\text { the first } \\
2 \mathrm{~h} \text { of HD } \\
\text { sessions. }\end{array}$} & \multirow{3}{*}{$\begin{array}{c}\text { - Body } \\
\text { composition: Lean } \\
\text { leg mass } \\
\text { - Physical } \\
\text { performance } \\
\text { - QoL: Physical } \\
\text { component } \\
\text {-Unknow Exercise }\end{array}$} \\
\hline & & $\begin{array}{l}\text { MLG: } \\
\mathrm{n}=16\end{array}$ & $56.2 \pm 12.5$ & $\begin{array}{c}11 \\
(40.74 \%) \\
\end{array}$ & & & & & \\
\hline & & $C: n=20$ & $56.9 \pm 12.4$ & $\begin{array}{c}0 \\
(0.00 \%) \\
\end{array}$ & & & & & \\
\hline \multirow[b]{2}{*}{$\begin{array}{c}\text { Martins et } \\
\text { al. [27] }\end{array}$} & \multirow[b]{2}{*}{ Brazil } & $\mathrm{I}: \mathrm{n}=12$ & $49.3 \pm 12.4$ & $\begin{array}{c}0 \\
(0.00 \%) \\
\end{array}$ & \multirow[b]{2}{*}{$\begin{array}{l}\text { lower limb } \\
\text { stretching } \\
\text { exercises }\end{array}$} & \multirow{2}{*}{$\begin{array}{l}\text { Weighted } \\
\text { ankle cuffs } \\
\text { and } \\
\text { dumbbells }\end{array}$} & $\begin{array}{l}\text { F: } 3 \text { times/week } \\
\text { I: } 10 \text { Rep. } \times 2-3\end{array}$ & \multirow{2}{*}{$\begin{array}{l}\text { Intradialytic: } \\
\text { the first } \\
2 \mathrm{~h} \text { of HD } \\
\text { sessions. }\end{array}$} & \multirow{2}{*}{$\begin{array}{c}\text { - Physical Activity } \\
\text { - Muscle strength } \\
\text { - Physical } \\
\text { performance } \\
\text { - QoL: Physical } \\
\text { component }\end{array}$} \\
\hline & & $C: n=12$ & $60.4 \pm 10.6$ & $\begin{array}{c}0 \\
(0.00 \%)\end{array}$ & & & $\begin{array}{c}\text { sets. } \\
\text { I: low intensity } \\
\text { (Borg's scale = } 3 \\
-5 \text { of } 10 \text { ) } \\
\text { T: } 12 \text { weeks. }\end{array}$ & & \\
\hline
\end{tabular}

*I = Intervention group, $\mathrm{C}=$ Control group,

** FIIT ( $\mathrm{F}=$ Frequency, I = Interval, I = Intensity, T = Times)

HLG = High-Load intradialytic Group, MLG = Moderate-load intradialytic Group, HD = Hemodialysis, QoL = Quality of Li 
Table 3 Display a meta-analysis of the 8-research publication (RCTs) of resistance exercise in end stage renal failure and hemodialysis patients including Figures 4 - 7.

Figure 4 Physical performance

Short Physical Performance Battery (SPPB)

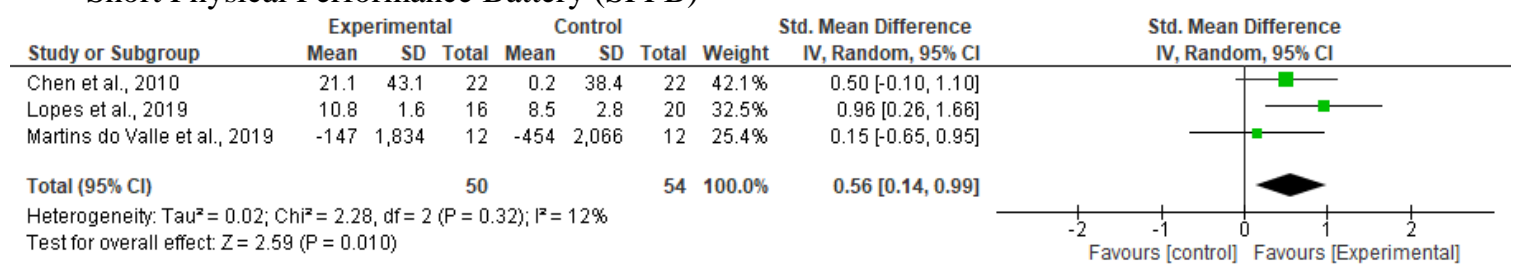

Strategic Test Solution (STS)

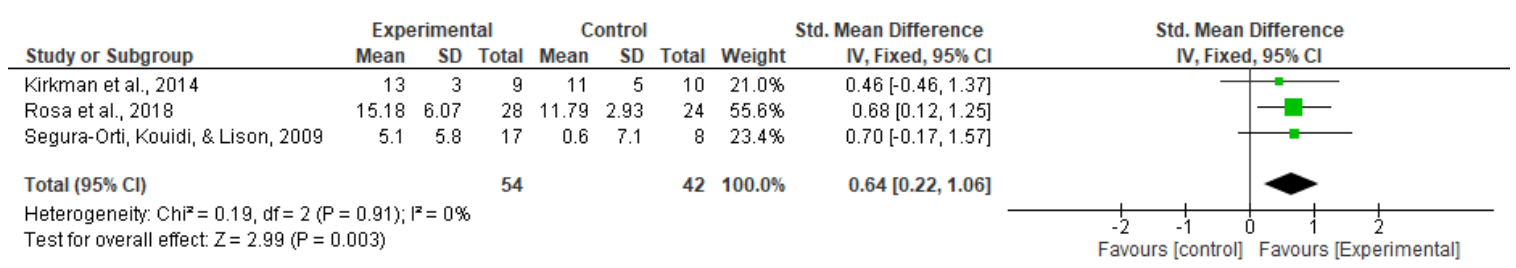

6-Minute Walk Test (6MWT)

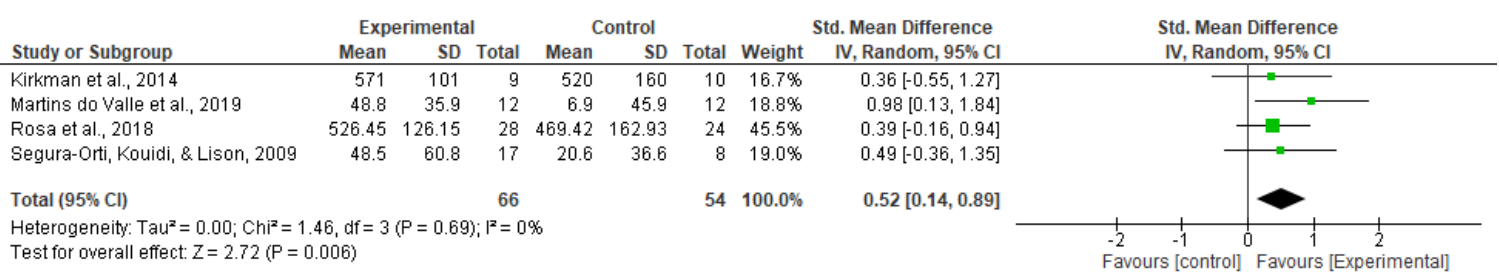

Figure 5 Quality of Life

SF-36 (Physical function)

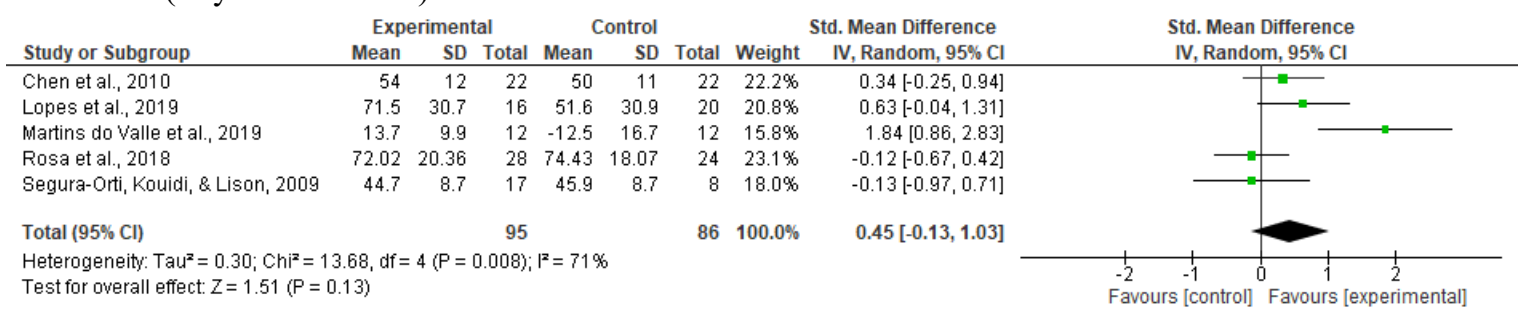

Figure 6 Muscle strength 
Handgrip strength

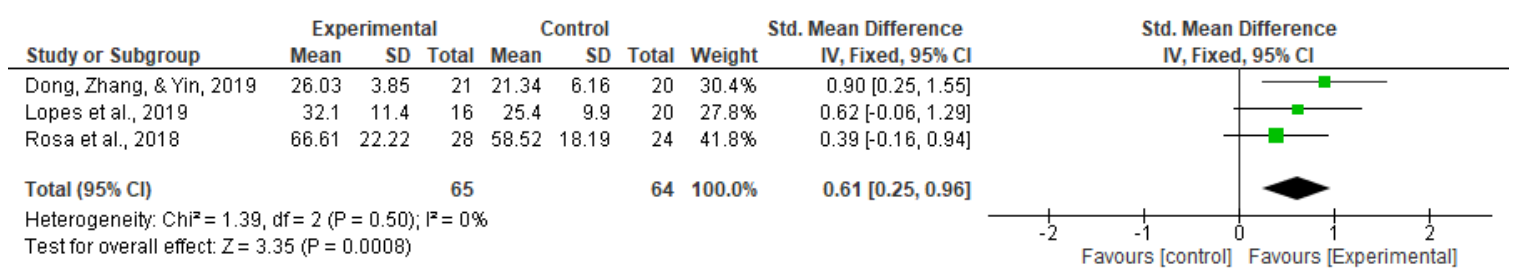

Knee extensor strength forest

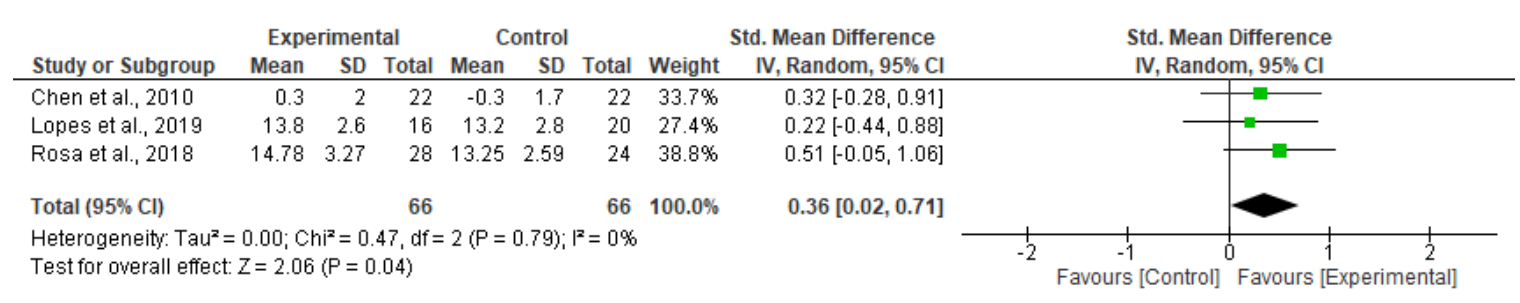

Figure 7 Body Composition

Leg lean Mass

\begin{tabular}{|c|c|c|c|c|c|c|c|c|c|c|c|}
\hline \multirow[b]{2}{*}{ Study or Subgroup } & \multicolumn{3}{|c|}{ Experimental } & \multicolumn{3}{|c|}{ Control } & \multicolumn{2}{|r|}{ Std. Mean Difference } & \multirow{2}{*}{\multicolumn{2}{|c|}{$\begin{array}{l}\text { Std. Mean Difference } \\
\text { IV, Random, } 95 \% \mathrm{Cl}\end{array}$}} & \\
\hline & Mean & SD & Total & Mean & SD & Total & Weight & IV, Random, $95 \% \mathrm{Cl}$ & & & \\
\hline Chen et al., 2010 & 15.8 & 5 & 22 & 12.1 & 6.1 & 22 & $71.8 \%$ & $0.65[0.04,1.26]$ & & 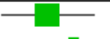 & \\
\hline Kirkman et al., 2014 & 287 & 86 & 9 & 201 & 77 & 10 & $28.2 \%$ & $1.01[0.04,1.98]$ & & & \\
\hline Total $(95 \% \mathrm{Cl})$ & & & 31 & & & 32 & $100.0 \%$ & $0.75[0.24,1.27]$ & & & \\
\hline \multicolumn{9}{|c|}{$\begin{array}{l}\text { Heterogeneity: } \operatorname{Tau}^{2}=0.00 ; \mathrm{Chi}^{2}=0.38, \mathrm{df}=1(\mathrm{P}=0.54) ; \mathrm{I}^{2}=0 \% \\
\text { Test for overall effect: } Z=2.86(P=0.004)\end{array}$} & $\begin{array}{ccc} & -2 & -1 \\
\text { Favours [control] }\end{array}$ & $\begin{array}{r}1 \\
\text { Favours }\end{array}$ & 2 \\
\hline
\end{tabular}

\section{Pooled results}

Table 3 (Figures 4 - 7) display a meta-analysis of the selected randomized controlled trials (RCTs). The study was able to combine the results of 8 studies in order to obtain the highest quality optimizing the results for future research. In sum, the 8 studies had 265 participants, divided into 131 participants who received an exercise program, and 134 participants in the control group. The authors assessed the effects of the programs on 4 outcomes: 1) physical performance, 2) quality of life (QOL), 3) muscle strength and 4) body composition.

\section{Effect on physical performance}

As shown in Figure 4, the researcher divided subgroups of influence on physical resistance exercise from different evaluation by applying Short Physical Performance Battery test (SPPB), Strategic Test Solution (STS) and 6-Minute Walk Test (6MWT) in direct order.

The first physical performance, which was evaluated by SPPB based on the influence of 3 RCTs $[14,26,27]$ with total sample size of 50 participants based on size heterogeneity. There was no significant difference between the studies $\left(\mathrm{X}^{2}=2.28, \mathrm{I}^{2}=12 \%, p=0.32\right)$ when the effect size was included between the resistance exercise program group and the control group. The result show that there was a significant in 2 groups $(\mathrm{SMD}=0.56,95 \% \mathrm{CI}: 0.14-0.99, p=0.010)$.

The second physical performance evaluated using STS score from the comparison of Influence rate for 3 RCTs [19,28,31]. The result show that a sample size of 54 participants based on a size diversity has no heterogeneity between the studies $\left(\mathrm{X}^{2}=0.19, \mathrm{I}^{2}=0 \%, p=0.91\right)$. when combine the effect size was included between the resistance exercise program and the control group. There was a significant $(\mathrm{SMD}=$ $0.64,95 \%$ CI: $0.22-1.06, p=0.003$ ).

The third physical performance that was evaluated by 6MWT to evaluate the difference of 4 RCTs influence rate $[19,27,28,31]$ with sample size of 66 participants based on size heterogeneity. The result consumes that there was no significant between the studies $\left(\mathrm{X}^{2}=1.46, \mathrm{I}^{2}=0 \%, p=0.69\right)$. However, when 
the effect size of the resistance exercise program group combines with the control group. There was a significant $(\mathrm{SMD}=0.52,95 \% \mathrm{CI}: 0.14-0.89, p=0.006)$.

\section{Effect on quality of life}

According to Figure 5 display the influence rate of resistance exercise on Physical component of QOL. By comparison of influence values of 5 RCTs [14,26-28,31]. In a sample size of 95 participants based on size heterogeneity. However, there was a significant different between the studies $\left(\mathrm{X}^{2}=13.68, \mathrm{I}^{2}=71\right.$ $\%, p=0.008)$. To combine the effect size of the resistance exercise program group and the control group. There was no significant $(\mathrm{SMD}=0.45,95 \% \mathrm{CI}:-0.13-1.03, p=0.13$ ).

\section{Effect on muscle strength}

According to Figure 6, the researcher divided subgroups of influence of muscle resistance exercise strength. The result showing the influence of resistance exercise on muscle strength. This was evaluated include: The first is Hand grip strength comparing 3 RCTs [26,28,30]. Applying on the sample size 65 participants based on heterogeneity assessments. There was no significant between the studies $\left(\mathrm{X}^{2}=1.39\right.$, $\left.\mathrm{I}^{2}=0 \%, p=0.050\right)$. When the effect size included between the resistance exercise program group and the control group. There was a significant (SMD $=0.61,95 \%$ CI: $0.25-0.96, p=0.0008)$, the second is Knee extensor strength was assessed by comparing the 2 RCTs $(14,19)$. Applying on a sample size 31 participants based on heterogeneity assessments. The result show that there is no significant during the study $\left(\mathrm{X}^{2}=0.38\right.$, $\left.\mathrm{I}^{2}=0 \%, p=0.54\right)$. Nonetheless, when the effect size was included between the resistance exercise program group and control group, there was a significant (SMD =0.75, $95 \%$ CI: $0.24-1.27, p=0.004$ ).

\section{Effect on body composition}

According to Figure 7 display the influence of resistance exercise on body evaluated by using Leg lean mass compared with 3 RCTs [14,26,28]. Applying in a sample size of 66 participants based on heterogeneity assessment. The result concludes that there was no difference between the studies $\left(\mathrm{X}^{2}=0.47\right.$, $\left.\mathrm{I}^{2}=0 \%, p=0.79\right)$. However, when the effect size was included between the resistance exercise program group and the control group. There was a significant (SMD $=0.36,95 \% \mathrm{CI}: 0.02-0.71, p=0.04$ ).

\section{Discussion}

This systematic review of literature found that resistance exercise can increase muscle strength, muscle mass and hemodialysis sufficiency $(\mathrm{Kt} / \mathrm{V})$, as well as reduce fatigue. Moreover, it increases the ability for patients to perform physical activities and functions, which leads to improved quality of life for end stage renal disease patients undergoing hemodialysis. In addition, the literature review and metaanalysis as a level 1 study (randomized controlled trials (RCTs)). From a total of 8 studies from 6 countries found using database searches, there were no trials conducted in Thailand, and the risk bias was usually low.

This study found that most resistance exercise was of low to moderate intensity, 3 times a week, with 20 - 30 min sessions performing progressive resistance training. AHA and ACSM guidelines suggest that health should be promoted and maintained in chronic renal failure patients by stimulating physical activity, including exercise to improve muscle strength. This exercise is recommended to be of moderate to high intensity, with 10 - 15 reps per set depending on the patient's maximum ability [32]. According to the results of this research, the optimum duration of exercise program is different for each individual patient. This is because the frequency of exercise varies from study to study. Research involving low to medium intensity resistance exercise 3 times per week showed that it may take up to 6 months for patients to improve and reach acceptable performance [31]. However, the resistance exercise programs that frequently applying 3 times a week, the duration of the program $8-12$ weeks will produce more efficient results. Most of the equipment used in the exercise is small in size, easy-to-use and still produce results in just 8 - 12 weeks. However, studies report that using larger equipment can improve muscle strength, reduce fatigue, and increase the physical activity of the patient [19,29]. One possible reason is each study used different intensity and exercise equipment. As well as different in determining the duration of the resistance exercise study. On the other hand, the intensity of the exercise must be considered the ability and character of the patient to implement the resistance exercise program.

Following our analysis of the influence of exercise on physical performance, assessed by SPPB, STS and 6MWT, we conclude that resistance exercise had a statistically significant effect on physical performance $(p<0.01)$. This shows that resistance exercise can improve the patient's ability to function, although different assessments were used for each study. In addition. resistance exercise has also been shown to increase muscle strength in patients undergoing hemodialysis by combining the effect sizes of 
programs on hand grip and knee extensor strength. It can be assumed that resistance exercise programs significantly improved patient muscle strength compared to control groups $(p<0.01)$. Therefore, patients undertaking resistance exercise programs have stronger muscles arising from the stimulation of the nervous system to secrete hormones such as cortisol and adrenaline, which in turn inhibit the release of Tumor Necrosis Factor Alpha (TNF- $\alpha$ ) and inhibit Cytokine secretion, thereby reducing the inflammatory process in the body. This will improve the body's ability to synthesize more proteins, reducing energy and protein depletion [17]. In addition, our results indicated that resistance exercise could increase leg muscle mass. This is caused by resistance exercise's effect on muscle mRNA and insulin, which increase the growth factor of the muscle. This results in stimulation of muscle fiber formation, muscle hypertrophy and promotes better circulation of muscle energy. Furthermore, it helps to build stronger and more efficient muscles, allowing patients to perform daily activities on their own [18,19].

However, even after analyzing the influence of resistance exercise on physical quality of life, it was found that there was no statistical difference between the resistance exercise program group and the control group $(\mathrm{SMD}=0.45,95 \% \mathrm{CI}:-0.13-1.03, p=0.13)$. This could have been because heterogeneity analysis found that there was a statistically significant difference between the studies $\left(\mathrm{X}^{2}=13.68, \mathrm{I}^{2}=71 \%, p=\right.$ 0.008). Nonetheless, only Martins do Valle et al. (2019) found a statistically significant influence of resistance exercise $(\mathrm{SMD}=1.84,95 \% \mathrm{CI}: 0.86-2.83)$.

\section{Conclusions}

This systematic review was based on 8 meta-analyses showing little overlap included individual RCTs, and provides the most comprehensive overview up to data on the effectiveness of resistance exercise interventions on improve physical performance, quality of life (QOL), muscle strength and body composition. Further high-quality research is needed, especially concerning onset of physical and psychological outcome, and the effects of sex, age, sign and symptoms (fatigue, mental functions), laboratory (albumin levels, Kt/Vs), and characteristics of exercise. Nonetheless, the evidence from this study suggests that resistance exercise effect on high physical performance, quality of life (QOL), muscle strength and body composition. As exercise has shown to be beneficial for many health aspects and is an inexpensive, easily modifiable lifestyle factor with virtually no negative side effects, the evidence from this meta-analysis might help to develop early intervention strategies for physical and psychological outcome both of patients receiving hemodialysis and other patients with similar chronic diseases.

\section{Recommendations}

\section{Suggestions for applying research results}

1) The results of this literature review can be used to develop a resistance exercise model suitable for end stage renal failure patients undergoing hemodialysis, as well as patients with similar characteristics.

2) The results of the literature review and effect sizes from meta-analysis can be apply in the development and design of resistance exercise programs for end stage renal disease patients undergoing hemodialysis, in order to develop and promote muscle mass building, muscle strength, ability to perform duties or functional conditions and better quality of life.

\section{Suggestions for further research}

1) Applying the results from the literature review and the influence magnitude from meta-analysis that were used in research studies in term of Intervention or program such as The effects of resistance exercise program among to end-stage renal failure patients receiving hemodialysis or other groups.

2) Comparing the results of this literature review and meta-analysis, in which exercise programs were supervised, with the effects of resistance exercise programs that patients undertake by themselves at home.

\section{Acknowledgments}

This research was supported by a 90-year scholarship from Chulalongkorn University. Ratchadaphisek Somphot Fund from the graduate school Chulalongkorn University, Thailand. 


\section{References}

[1] US Renal Data System. Annual data report: Atlas of chronic kidney disease and end-stage renal disease in the United States. National Institutes of Health, National Institute of Diabetes and Digestive and Kidney Diseases, Bethesda, MD, United States, 2013.

[2] Nephology Society of Thailand. Project: The study in progress and clinical outcome in patient with CRF in Thai SEEK. Nephology Society of Thailand, 1998.

[3] AC Webster, EV Nagler, RL Morton and P Masson. Chronic kidney disease. Lancet 2017; 389, 1238 52.

[4] M Jhamb, K Liang, J Yabes, JL Steel, MA Dew, N Shah and M Unruh. Prevalence and correlates of fatigue in chronic kidney disease and end-stage renal disease: Are sleep disorders a key to understanding fatigue? Am. J. Nephrol. 2013; 38, 489-95.

[5] S Senanayake, N Gunawardena, P Palihawadana, P Bandara, R Haniffa, R Karunarathna and P Kumara. Symptom burden in chronic kidney disease: A population based cross sectional study. BMC Nephrol. 2017; 18, 228.

[6] NG Kutner, R Zhang, Y Huang and P Painter. Gait speed and mortality, hospitalization, and functional status change among hemodialysis patients: A US renal data system special study. Am. J. Kidney Dis. 2015; 66, 297-304.

[7] SY Wang, XY Zang, SH Fu, J Bai, JD Liu, L Tian, YY Feng and Y Zhao. Factors related to fatigue in Chinese patients with end-stage renal disease receiving maintenance hemodialysis: A multi-center cross-sectional study. Ren. Fail. 2016; 38, 442-50.

[8] JC Kim, BB Shapiro, M Zhang, Y Li, J Porszasz, R Bross, U Feroze, R Upreti, K Kalantar-Zadeh and JD Kopple. Daily physical activity and physical function in adult maintenance hemodialysis patients. J. Cachexia Sarcopenia Muscle 2014; 5, 209-20.

[9] M Bossola, E Di Stasio, M Antocicco, G Pepe, L Tazza, G Zuccalà and A Laudisio. Functional impairment is associated with an increased risk of mortality in patients on chronic hemodialysis. $B M C$ Nephrol. 2016; 17, 72.

[10] AE Horigan. Fatigue in hemodialysis patients: A review of current knowledge. J. Pain Symptom Manag. 2012; 44, 715-24.

[11] KY Sohng and WJ Song. Effects of progressive resistance training on body composition, physical fitness and quality of life of patients on hemodialysis. J. Korean Acad. Nurs. 2012; 42, 947-56.

[12] R Bullani, Y El-Housseini, F Giordano, A Larcinese, L Ciutto, PC Bertrand, G Wuerzner, M Burnier and D Teta. Effect of intradialytic resistance band exercise on physical function in patients on maintenance hemodialysis: A pilot study. J. Ren. Nutr. 2011; 21, 61-5.

[13] YY Song, RJ Hu, YS Diao, L Chen and XL Jiang. Effects of exercise training on restless legs syndrome, depression, sleep quality and fatigue among hemodialysis patients: A systematic review and meta-analysis. J. Pain Symptom Manag. 2017; 55, 1184-95.

[14] JLT Chen, S Godfrey, TT Ng, R Moorthi, O Liangos, R Ruthazer, BL Jaber, AS Levey and C Castaneda-Sceppa. Effect of intra-dialytic, low-intensity strength training on functional capacity in adult haemodialysis patients: A randomized pilot trial. Nephrol. Dial. Transplant. 2010; 25, 1936-43.

[15] American College of Sports Medicine. ACSM's guidelines for exercise testing and prescription. Vol X. Wolters Kluwer, Philadelphia, United States, 2018.

[16] N Hongu, MJ Wells, PJ Gallaway and P Bilgic. Resistance training: Health benefits and recommendations. Univ. Arizona Coop. Ext. 2015; 21, 718-28.

[17] C Moraes, SM Marinho, AC da Nobrega, BDO Bessa, LV Jacobson, MB Stockler-Pinto, WSD Silva and D Mafra. Resistance exercise: A strategy to attenuate inflammation and protein-energy wasting in hemodialysis patients? Int. Urol. Nephrol. 2014; 46, 1655-62.

[18] M Saitoh, M Ogawa, MRD Santos, H Kondo, K Suga, H Itoh and Y Tabata. Effects of intradialytic resistance exercise on protein energy wasting, physical performance and physical activity in ambulatory patients on dialysis: A single-center preliminary study in a Japanese dialysis facility. Ther. Apher. Dial. 2016; 20, 632-8.

[19] DL Kirkman, P Mullins, NA Junglee, M Kumwenda, MM Jibani and JH Macdonald. Anabolic exercise in haemodialysis patients: A randomised controlled pilot study. J. Cachexia Sarcopenia Muscle 2014; 5, 199-207.

[20] MCD Lima, CDL Cicotoste, SD Kelly Cardoso, LA Forgiarini, MB Monteiro and AS Dias. Effect of exercise performed during hemodialysis: Strength versus aerobic. Ren. Fail. 2013; 35, 697-704.

[21] D Chan and BS Cheema. Progressive resistance training in end-stage renal disease: Systematic review. Am. J. Nephrol. 2016; 44, 32-45. 
[22] S Marinho, JC Eduardo and D Mafra. Effect of a resistance exercise training program on bone markers in hemodial ysis patients. Sci. Sports 2017; 32, 99-105.

[23] MCV Vilsteren, MHD Greef and RM Huisman. The effects of a low-to-moderate intensity preconditioning exercise programme linked with exercise counselling for sedentary haemodialysis patients in the Netherlands: Results of a randomized clinical trial. Nephrol. Dial. Transplant. 2005; 20, 141-6.

[24] RevMan. The nordic cochrane centre, the cochrane collaboration. RevMan, 2014.

[25] WG Cochran. Sampling techniques. Vol III. John Wiley \& Sons, New York, 1977.

[26] LCC Lopes, JF Mota, J Prestes, RM Schincaglia, DM Silva, NP Queiroz, ATVD Souza Freitas, FS Lira and MRG Peixoto. Intradialytic resistance training improves functional capacity and lean mass gain in individuals on hemodialysis: A randomized pilot trial. Arch. Phys. Med. Rehabil. 2019; 100, 2151-8.

[27] FMD Valle, BV Pinheiro, AAA Barros, WF Mendonça, ACD Oliveira, GDO Werneck and RBD Paula. Effects of intradialytic resistance training on physical activity in daily life, muscle strength, physical capacity and quality of life in hemodialysis patients: A randomized clinical trial. Disabil. Rehabil. 2020; 42, 3638-44.

[28] CSC Rosa, DY Nishimoto, GD Souza, AP Ramirez, CO Carletti, CGL Daibem, GK Sakkas and HL Monteiro. Effect of continuous progressive resistance training during hemodialysis on body composition, physical function and quality of life in end-stage renal disease patients: A randomized controlled trial. Clin. Rehabil. 2018; 32, 899-908.

[29] Y Chang, SY Cheng, M Lin, FY Gau and YFC Chao. The effectiveness of intradialytic leg ergometry exercise for improving sedentary life style and fatigue among patients with chronic kidney disease: A randomized clinical trial. Int. J. Nurs. Stud. 2010; 47, 1383-8.

[30] ZJ Dong, HL Zhang and LX Yin. Effects of intradialytic resistance exercise on systemic inflammation in maintenance hemodialysis patients with sarcopenia: a randomized controlled trial. Int. Urol. Nephrol. 2019; 51, 1415-24.

[31] E Segura-Orti, E Kouidi and JF Lison. Effect of resistance exercise during hemodialysis on physical function and quality of life: Randomized controlled trial. Clin. Nephrol. 2009; 71, 527-37.

[32] P Painter. Implementing exercise: What do we know? where do we go? Adv. Chron. Kidney Dis. 2009; 16, 536-44. 